\title{
How was the oral health of the older people in Tehran's Parks in 1396?
}

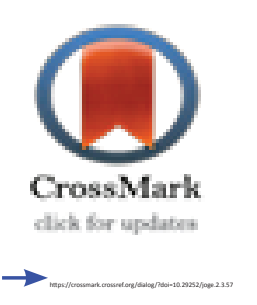

\section{*Farokhnezhad Afshar P1, Malakouti S.K², Ajri-Khameslou M³}

1- Tehran Psychiatric Institute, School of Behavioral Sciences and Mental Health, Iran University of Medical Sciences, Tehran, Iran (Correspondence Author)

E-mail: Pouyafarokhnezhad@yahoo.com

2- Tehran Psychiatric Institute, School of Behavioral Sciences and Mental Health, Iran University of Medical Sciences, Tehran, Iran.

3- Department of Critical Care Nursing, School of Nursing and Midwifery, Ardebil University of Medical Sciences, Ardebil, Iran.

\section{Abstract}

Introduction: Following the growth of the older population, attention to their health issues has become increasingly important. One of the issues that have been considered for various reasons is the oral health of the elderly. The purpose of this study was to determine the oral health status of older people in Tehran.

Method: This study is a descriptive and cross-sectional study. This study was conducted on 190 older people (113 old men and 77 old women) in Tehran's parks. The oral health status of the elderly was evaluated by GOHAI's oral health index, which has already been validated in Iran. Data were analyzed by mean, standard deviation and correlation and T independent test, using SPSS v.22 software.

Results: The mean age of participants was $67.30 \pm 6.87$. About $57.90 \%$ of the older adults did not have any of their teeth. The mean oral health of the older adults was $43.04 \pm 10.61$. There was a significant positive correlation between discomfort score and number of missing teeth $(\mathrm{P}<0.001$, $\mathrm{r}=0.51)$.

Conclusion: The results showed that the oral health status of the older adults is not appropriate in Tehran, which indicates the need to pay more attention to preventing oral problems and improving oral health.

Keywords: Aged, Oral Health, Quality of Life.

Received: 3/1/2018 Accepted: 18/2/2018

\begin{tabular}{|l|l|}
\hline \multicolumn{3}{|c|}{ Access this article online } \\
\hline
\end{tabular}




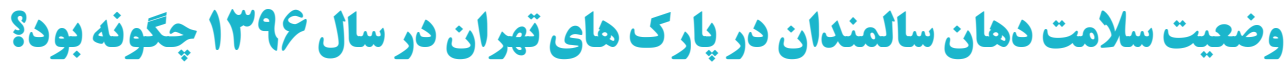

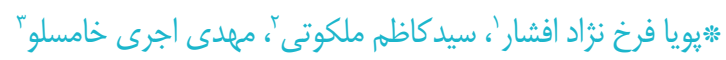

\author{
ا- انستيتو روانيزشكى تهران، دانشكده علوم رفتارى و سالمت روان، دانشكاه علوم يزشكى ايران، تهران، ايران (نويسنده هسئول)

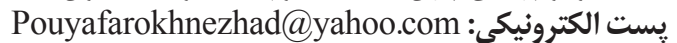

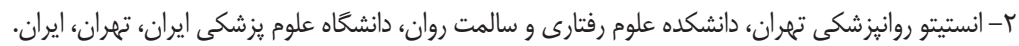

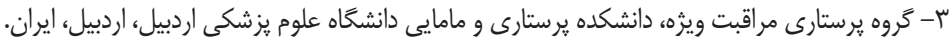

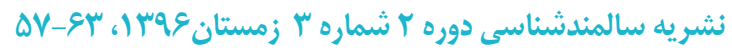

\footnotetext{
مقدمه: با رشد جمعيت سالمند، توجه به مسائل سلامتى آنان از اهميت روز افزونى برخوردار شده است. يكى از مسائلى كه به علل مختلف كمتر به آن توجه شده است سلامت دهان و دندان سالمندان است. هدف از مطالعه حاضر تعيين وضعيت سلامت دهان سالمندان در تهران مى باشد.

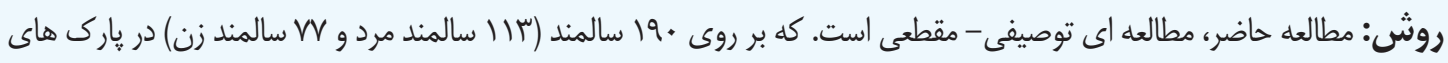

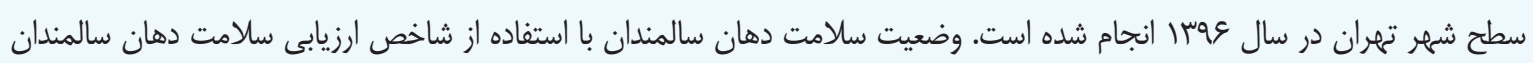

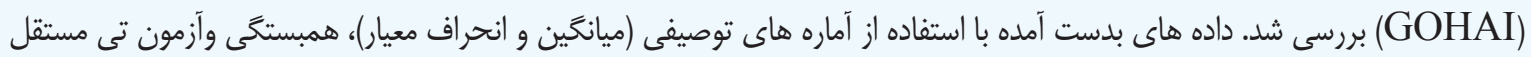

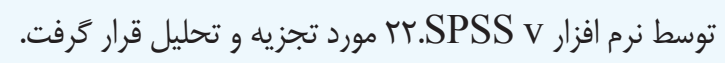

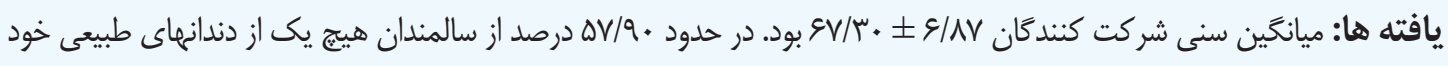

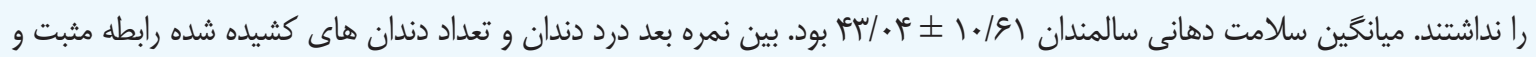

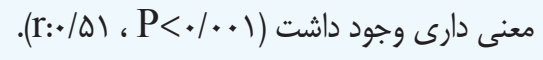

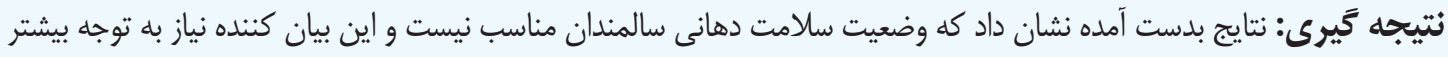

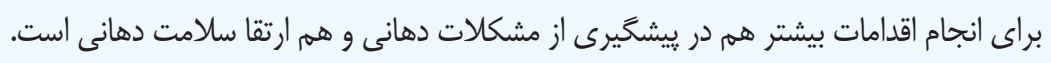

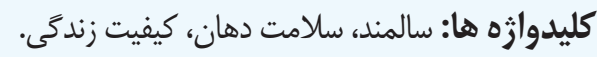


شده است كه بين سلامت دهان و دندان و ييشكَيرى از سكته قلبى

و سكته مغزى رابطه وجود دارد (9). مطالعات مختلفى در يزد، گَرَان و اصفهان در اين باره انجام

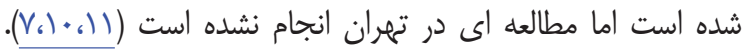
تاكنون مطالعات زيادى ييرامون سلامت دهان و دندان سالمندان

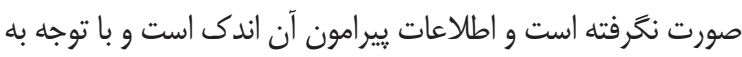
رشد جمعيت سالمندان بررسى هاى متعدد و دقيق مى تواند در بهبود

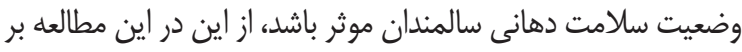
آن شديم كه به بررسى وضعيت سلامت دهان سالمندان بيردازيم.

\section{روش مطالكه}

اين مطالعه، مطالعه اى توصيفى- مقطعى با هدف تعيين وضعيت سلامت دهان سالمندان است. جامعل يزوهش كليه سالمندان در سطح يارك هاى شهر تهران بودند. على رغم اينكه نمونه كيرى

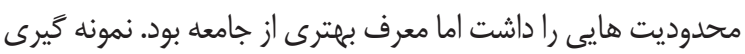

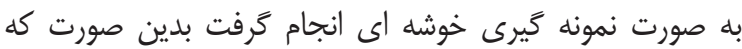

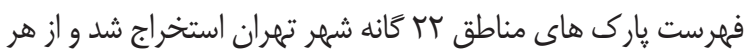
منطقه، يك يارك به تصادف انتخاب شد و نمونه كَيرى از سالمندان

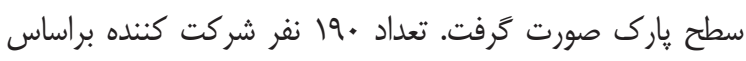
فرمول $n=\frac{\mathrm{Z}_{1-\frac{\alpha}{r}}^{r} \delta^{r}}{\mathrm{~d}^{r}}$

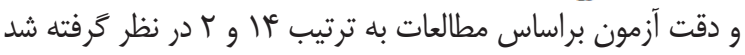
$n=\frac{196 \times 3 / 84}{4}=188(1 \cdot 61)$ معيارهاى ورود شامل: سن بيش از •9 سال، توانايى برقرارى ارتباط و همكارى، نبود بيماريهاى شناختى در سابقه شركت كنندكان

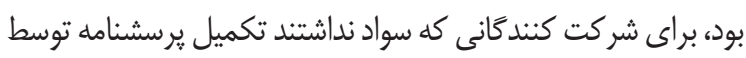
محقق به صورت مصاحبه انجام كَرفت. داده ها با استفاده از شاخص ارزيابى سلامت دهان سالمندان”

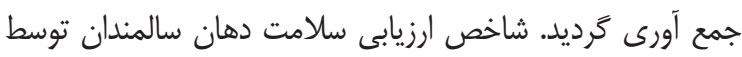

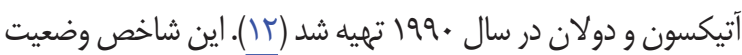
سلامت دهان سالمند را در سه ماه كَثشته مورد بررسى قرار مى دهد و مشتمل بر سه حيطه جسمانى (جهار سوال)، اجتماعى -روانى (ينج

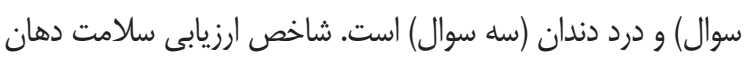
سالمندان داراى r ا سوال با ياسخ هاى طيف ليكرتى از هميشه (()،

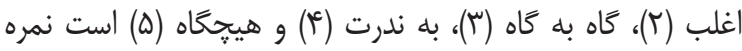
حاصل بين rا الى •و مى باشد و هرجه קيايينتر باشد نشان دهنده

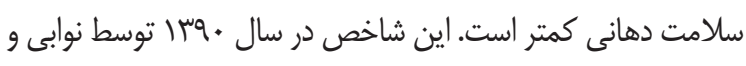

3- Geriatric Oral Health Assessment Index

\section{مقلهمه}

جمعيت سالمندان جهان به دنبال كاهش مرك و مير و مواليد از جندين دهه يِيش در حال افزايش بوده است و يِيش بينى

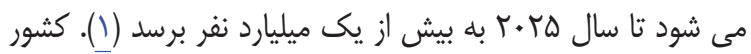
ايران نيز از اين مسئله به دور نبوده است و به دنبال بيشرفت هاى ليش يزشكى و فناورى هايى كه داشته است در حال سالخورده شدن است

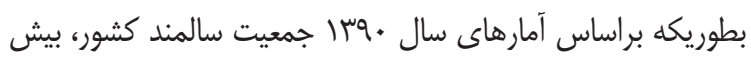

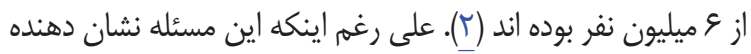
ييشرفت كشور در بسيارى از زمينه ها مى باشد اما نبايد از توجه به مسائل و مشكلات سالمندان غافل ماند. يكى از مشكلات بهداشتى

$$
\text { و سلامتى اين گروه سنى سلامت دهانى است. }
$$

سلامت دهان در تغذيه، سلامت ريوى، ارتباطات، تصوير از خود و عملكرد اجتماعى تاثير بسزايى دارد، از سوى ديخر بى دندانى باعث تاثير روى ظاهر و طرز صحبت مى شود و منجر به كاهش

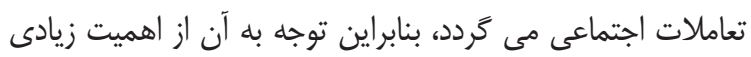

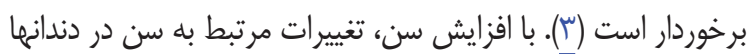
روى مى دهند كه شامل: ساييدگى و ترك برداشتن ميناى دندان، تحليل تاج دندان، سفت شدن عاج دندان، تحليل ريشه دندان، تحليل رفتن لثه ها و سمنتوم 'دندان مى باشد (أ)). از سوى ديخر سالمندان تمايل كمترى به استفاده از خدمات دندانيزشكى دارند و اين مى تواند ناشى از فقدان يوشش بيمه اى مناسب براى خدمات دندانيزشكى و

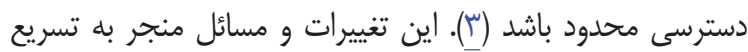

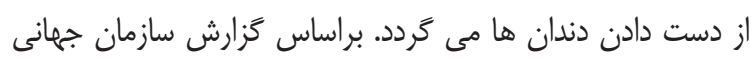

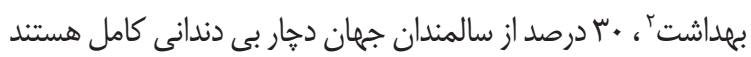

به نظر مى رسد عوامل مختلفى بر وضعيت دندانى سالمندان موثر مى باشند (مانند جنس، ميزان تحصيلات، وضعيت و غيره) (و). شناخت اين عوامل مى تواند مراقبين سلامت را در ارائه خدمات بهتر يارى كند. اثر سلامت بر كيفيت زندگى باعث توجه بسيار به مسائل مرتبط به سلامت شد، بيماريهاى مختلف مانند اختلالات دهانى بانى از طرق متفاوت بر كيفيت زندگى تاثير مى گذارند و با وجود اينكه مانكا

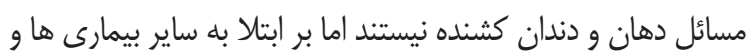
مسائل جسمى، روانى و اجتماعى مؤثر مى باشند (V). تاثير سلامت دهان بر سلامت عمومى و كيفيت زندگى به اندازه الى بود كه منجر

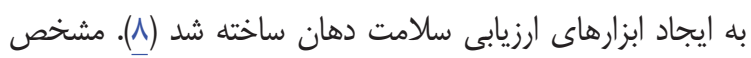

1- Cementum

2- World Health Organization 


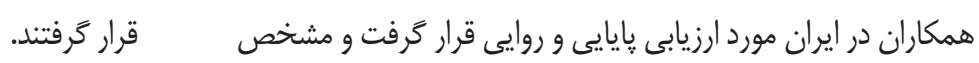

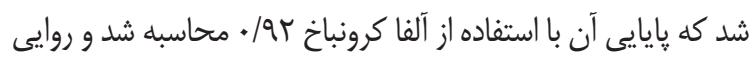

\section{كافقته هنا}

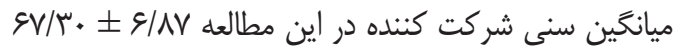

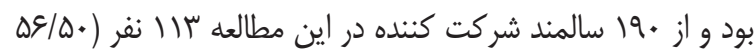
درصد) را مردان و WV نفر (•ه/N" درصد) را زنان تشكيل مى دادند. ساير ويثَيهاى دموگًرافيك در (جدول () نشان داده شده است.

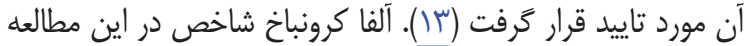

داده هاى به دست آمده با آماره هاى توصيفى (مانند: ميانخين،

انحراف معيار و فراوانى) و تحليلى (مانند همبستكى و آزمون تى

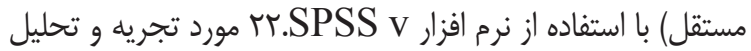

\begin{tabular}{|c|c|c|c|}
\hline \multicolumn{2}{|c|}{ فراوانى (درصد) } & \multirow{2}{*}{\multicolumn{2}{|c|}{ متغيرها }} \\
\hline زن & مرد & & \\
\hline$r(T / \varepsilon)$ & $\wedge(V / \Lambda)$ & مجرد & \\
\hline$\Delta V\left(V^{c}\right)$ & 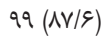 & متأهل & 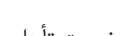 \\
\hline$r(r / q)$ & $r(T / V)$ & مطلقه & وصعيت نهل \\
\hline $10(19 / \Delta)$ & $r(\Gamma / V)$ & بيوه & \\
\hline$\Gamma \in(\mu \backslash / T)$ & $I T(1 . / 9)$ & بيسواد & \\
\hline$Y^{E}\left(\Gamma^{\top} / T\right)$ & (r)/q) & ابتدايى & \\
\hline$M(K \mu / \mu)$ & $r q(T \Delta / V)$ & سيكل & تحصيلات \\
\hline $9(11 / v)$ & rq (TQ/V) & متوسطه و ديبله & \\
\hline$r(r / \varphi)$ & $V(\xi / \pi)$ & دانشخاهى & \\
\hline$q(V / \Lambda)$ & $\wedge(V /))$ & بيكار & \\
\hline $9(11 / v)$ & $r \Delta(r q / \Lambda)$ & بازنشسته & \\
\hline$\Delta \cdot(95 / 9)$ & $1(\cdot / 9)$ & خانه دار & وضعيت اشتغال \\
\hline$r(r / q)$ & س & از كار افتاده & \\
\hline$q(11 / v)$ & $\notin q(F \cdot / V)$ & شاغل & \\
\hline
\end{tabular}

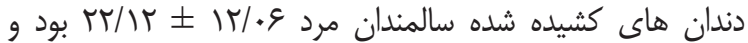

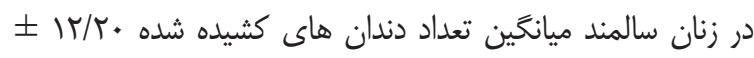

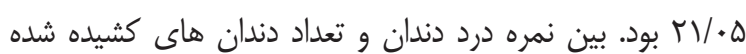

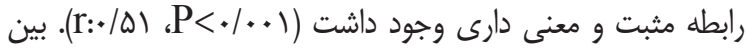
سئوال از خود ارزيابى از وضعيت دهان و دندان و نمره كل شاخص داري

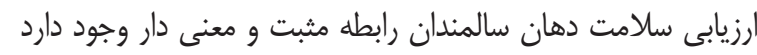

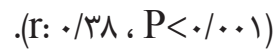

نمره سلامت دهانى سالمندان با سن رابطه معنى دارى را

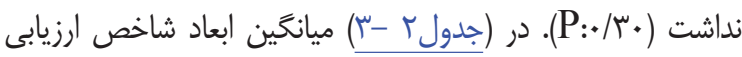
سلامت دهان سالمندان شركت كننده براى هر دو جنس و فراوانى إنى ياسخ هاى شركت كنندكان در مطالعه نشان داده شده است.
از بين •19 شركت كننده در اين مطالعه ا نفر (NT/9

درصد) اظهار كرده بودند كه فقط در زمان وقوع درد دندانى به

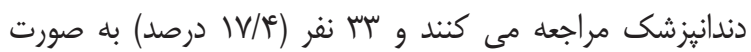
نامنظه براى دريافت خدمات دندايزشكى مراجعه مى كنند و هيج يك از شركت كنندكان مراجعه منظهم به دندانيزشكى نداشتند. از شركت

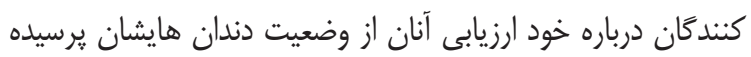

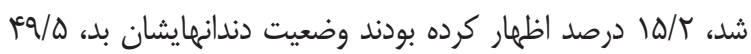
درصد متوسط، \&/Y درصد خوب، \&/ ا درصد خيلى خوب گزارش

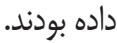
در حدود • |l نفر (ه درصد) از سالمندان، از دندان مصنوعى

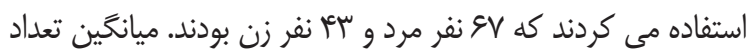

\begin{tabular}{|c|c|c|c|}
\hline $\begin{array}{c}\text { نتايج } 1 \text { Mf: } \\
\text { Df }\end{array}$ & زن & 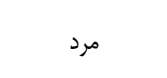 & جنس ا \\
\hline$P: \bullet / \Delta q$ & $|r / \Lambda| \pm r / \widetilde{\Delta}$ & $\| F / M \Phi \pm \varphi / \mathscr{q}$ & بعد جسمانى \\
\hline$P: \cdot / \Delta F$ & $W / F F \pm F / r r$ & $|N / \Delta \pm F / F|$ & بعد اجتماعي و روانى \\
\hline$P: * / V Q$ & $1 \cdot / v \omega \pm r / \cdot v$ & $1 \cdot|M \pm r / r|$ & درد دندان \\
\hline$P:-/ 97$ & $r v / q V \pm 1 . / T \Delta$ & $\mid \varphi r / \cdot \Lambda \pm 1 \cdot / \wedge q$ & كل \\
\hline
\end{tabular}


جدول س: فراوانى پاسخ ها به شاخص ارزيابى سلامت دهان سالمندان

\begin{tabular}{|c|c|c|c|c|c|}
\hline تعداد (درصداه (1) & تعداد (درصد) اغلب (T) & تعداه به كاه (در) & تعداد (درصد) & تعداد (درصداه (ه) & ياسخ ها \\
\hline & & & & & \multirow{13}{*}{ 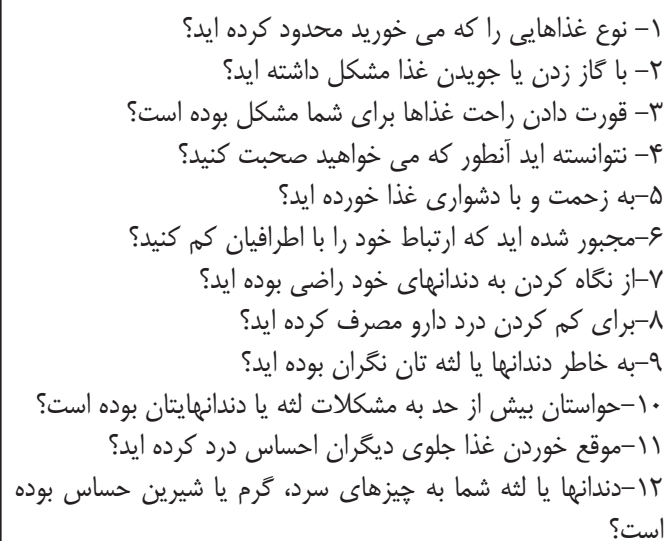 } \\
\hline RT (Tr/I) & $p q(r \Delta / \Lambda)$ & $19(\Lambda / F)$ & $r T(11 / 9)$ & 9) (rT/I) & \\
\hline$r(11 / 9)$ & $g V(\Gamma \Delta / \Gamma)$ & $r \Delta(I T / r)$ & سז & $\Delta T$ (TV/q) & \\
\hline $\operatorname{IV}(\wedge / 9)$ & $\|(\xi / \Lambda)$ & ऍ $\left(r_{*}\right)$ & سז (IT/I) & $99(\Delta r / 1)$ & \\
\hline$q(F / v)$ & $1 f(V / F)$ & $\Delta \cdot(r \varepsilon / \Gamma)$ & rT (1I/g) & $9 \Delta(\Delta \cdot)$ & \\
\hline $\operatorname{IV}(\Lambda / 9)$ & $\Delta \Delta(Y \wedge / Q)$ & ع (1N/q) & $\varepsilon(\Gamma / T)$ & $\operatorname{V\varepsilon }\left(x^{\kappa} \cdot\right)$ & \\
\hline$r(1 / 1)$ & $r \Delta(I T / T)$ & سז & $r \varepsilon(I T / V)$ & $\operatorname{lIf}(\varepsilon \cdot)$ & \\
\hline$\Delta \mathcal{L}(T N / \mathcal{F})$ & 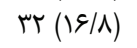 & (IT/V) & $r q(1 \omega / \mu)$ & $r q(r \Delta / \Lambda)$ & \\
\hline $1 \cdot(\Delta / \Gamma)$ & $\Gamma \Delta(\mid \Lambda / ז)$ & r $\left(r_{*}\right)$ & r (IE/A) & $V \Delta(r q / \Delta)$ & \\
\hline$V(\Gamma / V)$ & $r \cdot(r \backslash /)$ & $\Delta F(T N / F)$ & $\operatorname{IV}(\mathrm{N} / \mathrm{Q})$ & $V T(Y V / \Delta)$ & \\
\hline$\vee(r / v)$ & I (I\&/س) & $r \Delta(I T / T)$ & 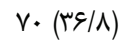 & $\Delta V(r \cdot)$ & \\
\hline$q(F / V)$ & $r \varepsilon(I T / V)$ & $r \Delta(T H / V)$ & $M F(I T / G)$ & $\Lambda \mathcal{S}(\mathcal{L} \Delta / \mu)$ & \\
\hline$M(9 / \Delta)$ & $19(1 \cdot)$ & $r \Delta(M / \mathcal{F})$ & 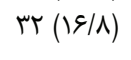 & $\Lambda \varepsilon\left(\Gamma^{\alpha} \Delta / \mu\right)$ & \\
\hline
\end{tabular}

كشيده شده دو برابر مطالعه ذكر شده بود (1). در مطالعه فائضى

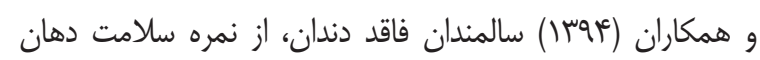

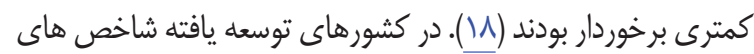
سلامت دهان سالمندان در حال بهتر شدن مى باشد، اين در حالى است كه اين شاخص در كشور ايران هنوز نامطلوب مى باشند (19). هيج يك از شركت كننداً مراجعه منظمه به دندانيزشكى نداشتند،

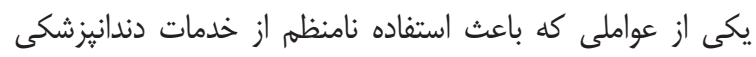

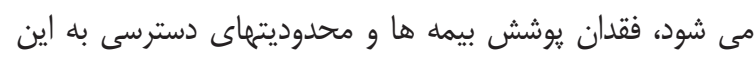

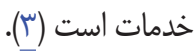

در حدود نيمى از سالمندان وضعيت دندان هاى خود را در

سطح متوسط كزارش كرده بودند و براساس همبستكى بالايى كه نمره شاخص ارزيابى سلامت دهان سالمند و خود ارزيابى فرد دارد

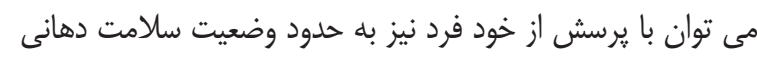

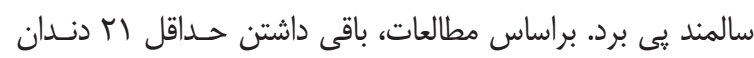
طبيعى در عملكرد طبيعى دهان و دندان لازم است و اين تعداد

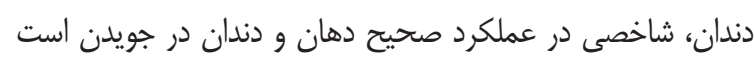

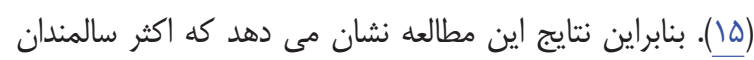
شركت كننده در مطالعه عملكرد صحيح دهانى -دندانى نداشتند. محدوديت هاى يزوهش شامل: تعداد اندك مطالعات يِيرامون

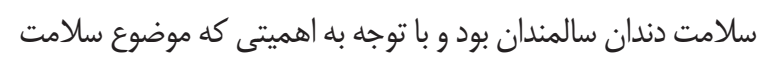
دهان و دندان دارد ييشنهاد مى شود بيشتر مورد توجه و تحقيق قرار

\section{تُنيجه تَيرى نهائى}

يافته هاى اين مطالعه حاكى از آن است سطح شاخص هاى سلامت دهانى سالمندان در جامعه در وضعيت نامطلوب قرار دارد
ايحث مطالعه با هدف تعيين وضعيت سلامت دهان سالمندان در

شهر تهران انجام شد. با توجه به نتايج بدست هه درصد از سالمندان

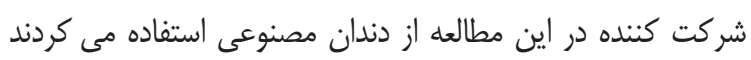

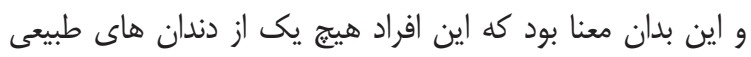

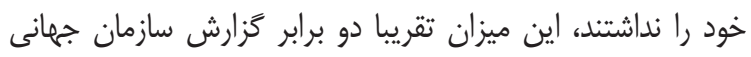

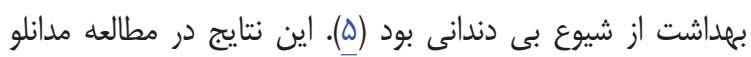

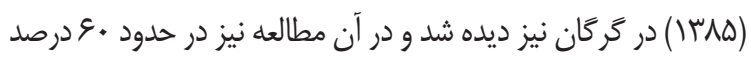
از سالمندان بدون دندان بودند (1)). در مطالعه رشيدى و همكاران

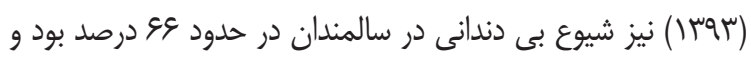

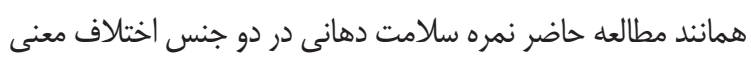

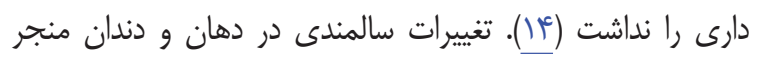

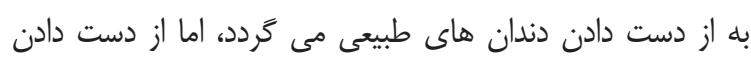
كامل دندانها إاتولوزيك مى باشد و اين فرد را مستعد تتذيه نامتعادل

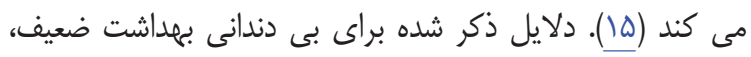

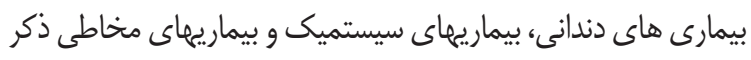

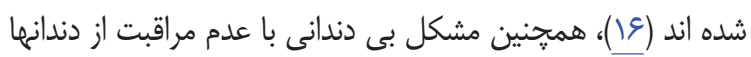

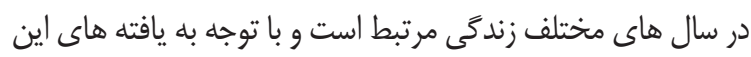

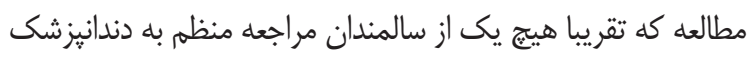

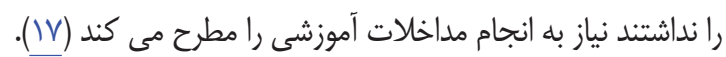

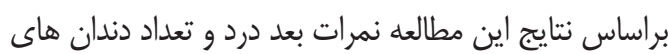

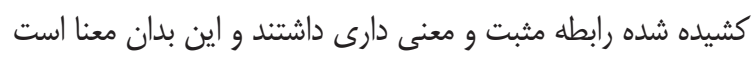

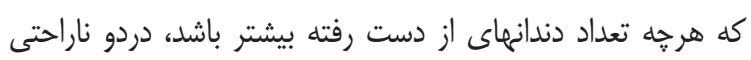

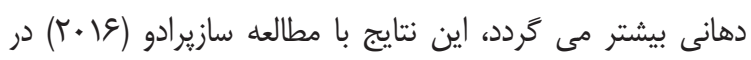

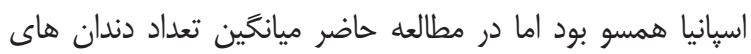


بود به طورى كه در حدود ه در دصد از سالمندان هيج دندانى نداشتند. بنابراين لازم است با مطالعات بيشتر ييرامون موضوع سلامت دهان و وردي دندان سالمندان، عوامل موثر را بيابيه.

\section{References}

1. Farokhnezhad Afshar P, Foroughan M, Vedadhir A, Ghazi Tabatabaie M. Psychometric properties of the Persian version of Social Adaptation Selfevaluation Scale in community-dwelling older adults. Clinical Interventions in Aging. 2017;12: 579-84.

2. Afshar PF, Asgari P, Shiri M, Bahramnezhad F. A Review of the Iran's elderly status according to the census records. Galen Medical Journal. 2016; 5 (1):1-6.

3. Fillit HM, Rockwood K, Young JB. Brocklehurst's Textbook of Geriatric Medicine and Gerontology E-Book: Elsevier Health Sciences; 2016.

4. Mauk KL. Gerontological nursing : competencies for care. First ed. Sudbury, Massachusetts: Jones \& Bartlett Pub; 2006.

5. Hajiebrahimi MH, Charkazi A, Rastgarimehr B, Homayonpour A, Hajiebrahimi Z, Mansourian $\mathrm{M}$, et al. ORAL HEALTH SITUATION IN ELDER PEOPLE IN GORGAN CITY, 2009. Iranian Journal of Diabetes and Lipid Disorders. 2014; 13 (6): 505-12.

6. Namal N, Can G, Vehid S, Koksal S, Kaypmaz A. Dental health status and risk factors for dental caries in adults in Istanbul, Turkey. Eastern Mediterranean Health Journal. 2008; 14 (1):110-8.

7. Haerian A, Sharifabadi M, Ali M,
و بايد اقداماتى در جهت يِيشَيرى از مشكلات دهانى و دندانى و

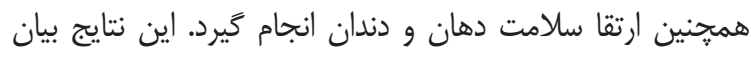
كننده هشدارى براى توجه ناكافى به مقوله سلامت دندان سالمندان

Amirian E. Relationship Between Oral Health Related Quality of Life and Dental Condition in Patients Referring to Yazd Dental University and Yazd Khatamolanbia Clinic. Journals of Community Health Research. 2015; 4 (2):105-13.

8. Sáez-Prado B, Haya-Fernández M-C, Sanz-García M-T. Oral health and quality of life in the municipal senior citizen' s social clubs for people over 65 of Valencia, Spain. Medicina oral, patologia oral y cirugia bucal. 2016; 21(6):672-8.

9. Series AS-CC, D'Aiuto F, Hingorani A, Smeeth L. Invasive Dental Treatment and Risk for Vascular Events. Ann Intern Med. 2010;153: 499-506.

10. Hajiebrahimi MH, Charkazi A, Rastgarimehr B, Homayonpour A, Hajiebrahimi Z, Mansourian M, et al. ORAL HEALTH SITUATION IN ELDER PEOPLE IN GORGAN CITY, 2009. Iranian journal of Diabetes and Metabolism. 2014; 13 (6): 505-12.

11. Modanloo M, Khosravee H, Ghobadee K, Abdollahi H, Ziaea T, Behnampour N. Dental health status in elderly (GorganIran). Journal of Gorgan University of Medical Sciences. 2010; 12 (3).

12. Atchison KA, Dolan T. Development of the geriatric oral health assessment index. Journal of dental education. 1990; 54 (11):680-7. 
13. Navabi N, Salahi S, SHariatmadar ahmadi A. Assessment of Oral Health Assessment Index (GOHAI) Validity in Iranian Elderly Population. journal of research in dental sciences. 2012; 9 (3):161-9.

14. Rashidi-Maybodi F, Haerian-Ardakani A, Zarabadi Pour M, Heydari-Postakan R, Pourbaferani H. Evaluation of Oral Health of Elderly Patients Referring to Khatam ol Anbia Clinic in Yazd in 2014. Journal of Health. 2016; 7 (2): 227-35.

15. Rabiei M, Shakiba M, Masoudirad H, Javadinia A. Dental status among urban and rural elderly of Talesh (2009). The Journal of Qazvin University of Medical Sciences. 2011;15 (3): 69-75.

16. Gosney M, Harper A, Conroy S. Oxford desk reference: geriatric medicine. 1th ed. Oxford: Oxford University Press; 2012. 596 p.

17. Kosari M, Hoseini Z, Golrizi F. The investigation of oral and dental status and its related factors in the elderly in Torbat Heydariyeh in 2015. Journal of Torbat Heydariyeh University of Medical Sciences. 2016; 4 (2): 39-44.

18. Faezi M, RejehN, Soukoti M.Assessment of oral health in older people referred to selected dentistry schools of universities of Tehran. Journal of Health Promotion Management. 2015; 5 (1):1-10.

19. Taraghi Z, Meskini L, Fani Saberi L. Oral Health on cognitive decline in the elderly. Clinical Excellence. 2016; 5 (2): 57-67. 